\title{
The views of mental health manager towards the use of a family work model for psychosis in Guangzhou, China
}

Article

Accepted Version

Dixon, J., Lei, J., Huang, W., Sin, J. and Smith, G. (2018) The views of mental health manager towards the use of a family work model for psychosis in Guangzhou, China. Community Mental Health Journal, 54 (7). pp. 1057-1063. ISSN 0010-3853 doi: https://doi.org/10.1007/s10597-018-0247-7 Available at https://centaur.reading.ac.uk/75663/

It is advisable to refer to the publisher's version if you intend to cite from the work. See Guidance on citing.

To link to this article DOI: http://dx.doi.org/10.1007/s10597-018-0247-7

Publisher: Springer

All outputs in CentAUR are protected by Intellectual Property Rights law, including copyright law. Copyright and IPR is retained by the creators or other copyright holders. Terms and conditions for use of this material are defined in the End User Agreement.

www.reading.ac.uk/centaur 
Central Archive at the University of Reading

Reading's research outputs online 
The Views of Mental Health Manager towards the Use of a Family Work Model for Psychosis in

\title{
Guangzhou, China
}

\begin{abstract}
Family Interventions in Psychosis (FIP) have been promoted internationally but have been criticised for being based on western cultural models. This paper reports on a focus group study with 10 Integrated Mental Health Service Managers in Guangzhou, China using thematic analysis. Managers believed FIP might benefit families but identified potential difficulties due to a) families avoiding services due to the 'shame' of mental illness b) unrealistic expectations of services amongst families c) deferral to 'key decision-makers' within families when discussing family issues with workers. The findings indicate that FIP work should focus on interaction between carers in the first instance with service users being introduced into sessions at a later date and that more attention needs to be given by the research community to how FIP may be adapted to cultural norms within China.
\end{abstract}

\section{Keywords}

Family Interventions; Family Therapy; Psychosis; Social Work in China; Qualitative Research 


\section{INTRODUCTION}

Psychosis can have significant effects on the lives of individuals and their families. Since the mid-2000s policy makers have recommended family interventions for psychosis (FIP) as a first line treatment, to be delivered in conjunction with medication and community support (Buccie et al., 2016). Attempts have been made to apply FIP to China with a systematic review by Pharaoh et al. (2010) reporting 28 trials since 1994. The Chinese government's five year mental health plan aims to develop rehabilitation services through integrating community and family support (National Health and Family Planning Commission of China, 2015). FIP may provide an effective means to offer rehabilitation at a community levels. However, thought needs to be given about whether FIP can accommodate current cultural models and whether it can be integrated into service designs (Chakrabati, 2011). We begin by considering current forms of FIP before going on to consider issues around adaption to China.

All FIP models focus on building alliances with family members, although the format of interventions vary. Broadly speaking FIP models are either therapeutically or psycho-educationally orientated (Pitshel-Walz et al., 2015). Therapeutically focussed models are facilitated by workers who provide education but also aim to use the family as a resource. For example, families may be encouraged to come together as a group in order to develop a shared understanding of psychosis drawing on biopsychosocial models.. They may also be encouraged to reach agreement about problems, identify solutions and review these in future meetings (Smith et al., 2007). Western therapeutic practice often treats the person with psychosis as an 'expert by experience' (Falloon et al., 1984) with approaches focussing on definitions of recovery voiced by users (Glynn et al., 2006). Models that are psychoeducationally focussed place emphasis on the family receiving information. Here relatives may be educated in groups without the person with psychosis being present. This form of education may provide a biomedical view of mental disorder or may draw on biopsychosocial models to explain factors relevant to relapse. Information may be simply received by families or used as the basis to problem-solve collectively (Chien et al., 2005). Both types of intervention are, in part, informed by theories of expressed emotion (EE) which are understood as behaviours from family members that are over-critical or over-protective. These were associated with increased rates of relapse in initial versions of the theory (Brown et al, 1958). Whilst previous interventions aimed to assess and reduce EE, models have now evolved to place greater emphasis on empathic engagement with the family through psychoeducation (MacFarlane et al., 2016; Sin et al., 2017). Developing family problem-solving skills through this approach has now been shown to reduce rates of relapse equal to that through reducing rates of EE (McFarlane, 2016). 
The majority of discussions about applying FIP to China have focussed on the concept of EE. Researchers have noted that EE was formed with English cultural norms in mind, valuing the independence of the person with psychosis (Singh, 2013). In contrast, Chinese culture has traditionally placed emphasis on interdependence (Lam et al., 2015). Chinese parenting practices can be seen to be guided by the notion of 'guan', which indicates a willingness for parents to direct their child through control and regulated teaching. Rather than being perceived as domineering, this approach has been viewed as 'organizational', valuing sacrifice by parents (Chao and Sue, 1996). Critics have questioned whether notions of EE focussing on 'over-involved' or 'over-critical' behaviours can be fruitfully applied within China and have proposed adapted measures (Singh, 2013). However, Chinese culture may also affect engagement with FIP. Beliefs that mental illness is related to the behaviour of ancestors or poor family dynamics may discourage help-seeking (Phillips et al., 2002). Even where medical models of disorder are accepted families may be reluctant to engage with services as it is common to place value on 'unity' which may discourage discussion of problems with outsiders ( $\mathrm{Li}$ and Chui, 2016). Consideration therefore needs to be given as to how these issues may be addressed within FIP.

Whilst FIP has been promoted by policy makers in developed countries, its application has often been limited due to organisational difficulties or professional resistance (Buccie et al., 2016). Different challenges are likely to apply in China influenced by service developments. Since 2004, the Chinese Government has placed emphasis on community services within mental health policy (Wong et al., 2014). Despite this, the majority of funding continues to be directed towards hospital-based services. Although individuals with mental health problems may admit themselves to hospital or be admitted compulsorily under the Chinese Mental Health Act 2013, the financial costs of admission remain high, even to those with medical insurance (Montgomery et al, 2013). Consequently, the majority of care continues to be provided by families. Recent policy has led to the development of mental health centres, whose workers are tasked with registering, risk assessing and offering community care to those with a severe mental disorder (Wong et al, 2014). Whilst these centres represent an expansion of provision, the amount of trained workers remains low. Consideration therefore needs to be given to whether the workforce believes FIP is viable and whether it can be integrated into service structures. The aim of this paper is to examine the views of managers of Integrated Mental Health Service Centres (IMHCs) to applying a FIP model to Guangzhou, China. The paper reports on a pilot study in which managers were asked about their views of applying FIP. The research identifies potential opportunities and difficulties in applying FIP to China. These data are used to suggest ways in which FIP might be adapted and areas for future research. 


\section{METHOD}

The study adopted a qualitative approach in order to explore the views of IMHC managers. The research was conducted in May 2015 in Guangzhou. The city has expanded its community mental health services in recent years as well as adopting public health programmes aimed at improving attitudes to mental illness. At the time the research was conducted there were 12 geographic districts in Guangzhou of which 11 had established their own Integrated Mental Health Service Centres (IMHSCs). IMHSCs were first launched in 2013 with the aim of providing crisis intervention and counselling services for community patients. IMHSCs in Guangzhou are sponsored by public funding and are run by a variety of Non-Governmental Organizations who have acquired contracts through competitive tendering.

Managers from IMHCs were invited to listen to a presentation about FIP and to take part in a subsequent focus group. All participants were sent an information sheet about the project by [author 2] and were invited to discuss the project. Of the 11 invited, all agreed to take part with 10 attending on the day. Seven of the participants were female and three male. Seven were employed as junior social workers and 3 as senior social workers. The length of time that participants had worked in mental health services ranged from 1-3 years with the majority $(n=6)$ having worked for 1 year in mental health services. The presentation and focus group was held at [named University]. A one hour presentation was given to participants about FIP. This included an overview of FIP, alongside explanations of EE and models of assessment. Participants were presented with examples of therapeutically orientated models and psycho-educational models. The focus group was facilitated by [author 1] and observed by [author 2]. A semi-structured guide was used based around research questions written by [author 1] with input from [author 4]. A professional interpreter was used to interpret questions from [author 1] and to translate participants' discussion. Participants were asked what they saw as the main needs of individuals with psychosis and their families. They were also asked how they felt that psychological support was received by families. Participants were asked for their opinions as to whether an FIP model was congruent with Chinese values. Finally they were asked about whether they felt FIP could be meaningfully applied in their areas and if so how this might be facilitated.

Focus group were recorded and transcribed into Cantonese. [Author 2] and [author 3] coded transcripts independently identifying key themes through thematic analysis (Braun and Clarke, 2006). Coders reached similar 
conclusions about themes, so there was no need to involve a third coder to resolve differences. Names for key themes and key quotations were agreed and were translated into English by [author 2]. The researchers adopted a constructionist position toward the data, being mindful that what counts as valid knowledge within a group is historically formed and constantly negotiated. Focus groups were treated as a site in which tacit knowledge could be "generated, maintained or changed" and through which participants could, "explore opinions, beliefs and understandings about a program or policy...through a form of collective sense making” (Ryan et al., 2014: 1). The research received ethical approval from [University ethics committee] in May 2015. All participants gave signed consent. Pseudonyms have been used throughout. There are no known conflicts of interest.

\section{RESULTS}

Attitudes of Family Members toward Mental Health Support and Treatment

In thinking about whether FIP might be offered to families in Guangzhou, participants considered issues around engaging with families generally. As managers of IMHCs, participants were tasked with registering the mental health needs of those in their area and offering support. However, this process was rarely straightforward. Managers noted that individuals and families often failed to respond to requests to register. This reluctance was attributed to the cultural stigma attached to mental disorder. Participants highlighted that families commonly believed that people with mental health problems were 'incapable' or 'handicapped' and could not be treated. Furthermore, stigma was seen to affect the whole family. Participants gave several examples in which families had hidden a family member’s mental illness from community members. Ms Ye stated:

The Chinese believe in this saying, 'don't wash your dirty linen in public'. This is deeply rooted in our culture.

The family are so ashamed...they don't want others to know that they have a 'crazy' member in the family.

The opinion above was supported by an example in which a family had admitted a relative to psychiatric hospital but explained this to members of the community by telling them that he had gone to care for a sick relative. Similar examples were given by other participants in which families hid mental health problems to avoid stigma. These issues were perceived to be more common amongst middle class families who were seen to be more susceptible to public shame. 
Nonetheless, some families did come forward for help. Participant related that families would often express high levels of emotion in the first few sessions with workers. Whilst this presentation was felt to be a true reflection of distress, participants felt that families presented with particular priorities in mind. Two inter-related themes emerged. First, presenting families were seen to be concerned about the economic impact of caring for a family member. Participants indicated that whilst some drug treatments were provided by the state other drug costs had to be provided by the family. In addition families were further burdened when the person suffering from a mental health problem could not work. An expectation amongst presenting families was that mental health workers would enable them to access practical assistance such as government grants or aid. For example, Mr. Zhang stated that,

They usually want us to relieve their economic difficulties, especially in relation to applying for government supports.

Participants indicated that whilst other services would be provided by IMHCs, family concerns about finances would continue to frame interventions with families being more likely to withdraw from services if this issue could not be resolved. Second, participants felt that families saw recovery from illness as a medical outcome. By contrast, psychological support or counselling tended to be viewed as time-consuming and ineffective, even though counselling was provided by IMHCs for free. From the perspective of research participants, families usually had a high expectation of what might be offered in terms of treatment. Furthermore their enthusiasm would hugely decrease if they could not perceive any 'practical' (Mr. Zhang), 'effective' (Ms. Chen), 'improving' or 'enabling' (Ms. Dong) results.

\section{Cultural Norms within Families}

Participants indicated a number of cultural norms within families in their area that would be relevant were FIP to be offered. Three themes emerged. First, participants indicated the importance of identifying 'key decision makers' within families. Much of the literature on Chinese families has focussed on the cultural value of obedience to parents. Whilst participants did not disagree with this they indicated it was usual for one family member to have the most influence and that it was important to identify this person early on. Several research participants indicated that the person who earned the most money within the family had the most say. However, other members indicated circumstances in which this principle might be disrupted. Migration was seen to be one such 
circumstance. Here, parents might work in a different province leaving grandparents to care for the child. In these cases it was more difficult to identify key decision makers. In addition, one participant indicated that both parents might be present but that neither would be willing to make a decision leaving an uneasy dynamic. Second, participants highlighted cultural norms about obedience to authority figures. Participants indicated that whilst family members might view the actions of authority figures as insensitive, they would be reluctant to challenge them due to cultural norms. Mr. Zhang explained:

I think this is determined by the Chinese culture. Family members - especially when they are the children in a family - tend to have a higher level of tolerance or obedience to authority figures.

Several examples of parents referring to their children as 'stupid' or 'crazy' in front of mental health workers and other family members were given to support this. Third, participants indicated that families in their area were concerned to maintain an image of unity. Drawing on an example of a decision maker stating that their relative was 'stupid', participants felt that family members might react in one of two ways. First, members might accept that the family member was stupid because the decision maker was saying it. Second, members might disagree with this view, but would be reluctant to voice it. According to participants, even when family members did have a different opinion to the authority figure, they would 'try to maintain an atmosphere of harmony' (Mr. Zhao) and ‘avoid accelerating the conflicts’ (Ms. Wang). Ms. He supported this view stating:

In the family, I don't think other people will point out the mistakes of the decision maker in front of everyone; they would rather have a private communication [with the social worker] in order to save face.

A minority of participants felt that some families might speak out on occasions where a decision maker was seen to be particularly hostile to the person with a mental health problem, but these instances were seen as exceptional.

\section{APPROPRIATENESS OF FIP TO GUANGZHOU}

Participants believed that FIP could be applied to Guangzhou but identified several problems that might occur during implementation. An issue that was raised by several participants was whether the person with mental health problems should be involved within the FIP process. No consensus on this issue was reached, although 
some participants expressed doubts as to whether family members could be encouraged to listen to this person. This scepticism arose from common views that those with mental health problems were 'incapable'. Consequently, a number of participants felt that the person with mental health problems could not be involved until FIP sessions had been offered to other family members. Furthermore, some participants voiced scepticism as to whether it would be possible to apply a model where the views of all family members were given equal weight.

Several participants felt that an FIP model was unlikely to be effective in cases where a family member had experienced a mental health problem for many years. This view was based on the belief that that family members would have 'given up' and would be reluctant to engage. Whilst the majority were pessimistic about engaging with families, three participants indicated that FIP might appeal to some for financial reasons. They indicated that in the absence of finding an effective treatment, families would adopt the cheapest option. As IMHCs were able to offer free psychological treatment, these participants felt that this might be used as a means to engage families. These participants suggested that engagement could be furthered if IMHCs were able to offer financial incentives, such as travel expenses or a cash reward. All participants were optimistic about applying FIP where an individual had suffered from a first episode of psychosis. This was based on the views that families would be more receptive because they had not yet had a negative experience of treatment. For example, Ms. Xu stated that family members would be more willing to engage in FIP as, "they still have high motivation to change the situation at any cost"; a position that was accepted by the majority of the group.

\section{DISCUSSION}

Whilst there has been a growing literature assessing the effectiveness of FIP within China, there has been little discussion of what the challenges of implementing a service may be. The findings from our study indicate that there is a need to explore cultural and organisational factors to maximise benefits to families. A key theme raised by participants was the need to consider the way in which families engaged with mental health services generally. Mental health stigma was viewed as key. Stigma is generally understood to be "an attribute that is deeply discrediting” which makes a person less human or desirable to others (Goffman, 1963: 13). Mental health stigma can be experienced at an internal level (through shame experienced by the sufferer), at a group level (including families) or may be enacted at an institutional level through discriminatory laws and policies (Livingston and Boyd, 2010). Whilst participants in our research acknowledged individual stigma, group stigma was the factor 
seen to influence engagement the most. These findings confirm what is already known about mental health stigma in China, namely that Chinese families suffer from a particularly acute form of group shame. This sense of shame is based on the belief that illness highlights fault within family units leading to a loss of 'face' (Yang and Kleinmann, 2008). Strategies aimed at engaging families in FIP therefore need to consider how stigma may be played out within communities and may need to provide a level of community education to combat such beliefs.

FIP commonly aims to engage with families through addressing their presenting concerns. Here two themes emerged. The first was in relation to financial assistance, which were consistent with accounts of Chinese carers elsewhere (Ramsay, 2010; Li and Chui, 2016). In these studies carers identified that caring posed significant burdens due to the cost of treatment and because the person with a mental health problem was often not able to earn. In Guangzhou,, a previous study has found that $70 \%$ of carers had no medical insurance (Pearson, Paul and Lam, 2002). This may explain why financial issues were viewed as a priority by managers. The second theme related to the belief by participants that families sought a medical solution. Research elsewhere has found that the families of people with schizophrenia in China tend to seek a range of treatments during the early stages of psychosis in the hope that it can be treated through procedures such as an operation (Phillips, 1993). Furthermore psychiatric services tend to be sought as a last resort (Li et al., 2014). Even where individuals are seen by mental health services, families may remain sceptical of biomedical explanations. For example, a questionnaire study by Phillips et al (2000) which asked family members to rate reasons for their relative’s psychosis found that they gave preference to social, interpersonal and spiritual explanations over biomedical ones, even after the relative had been seen by a psychiatrist. These patterns suggest that families remain sceptical towards psychiatric knowledge which may account for their willingness to disengage with services where an improvement is not quickly forthcoming.

The majority of mental health research focussing on family dynamics in China focusses on the concept of EE. As stated earlier, there has been debate as to how useful this notion is within China with some authors suggesting adjusted measures. Although participants in our research were given information about EE, this did not arise as a major theme. However our research identified new findings in relation to patterns of caring within families. Traditionally, Chinese culture has afforded the most authority to parents. Rapid modernisation policies within China have been led to changes in family structure and attitudes to parental authority. For example, Li and Chui's 
(2016) research in a rural area found that siblings tended to focus on their own nuclear family leaving the burden of care to parents. Our research identified a different pattern caused by factors such as the absence of parents due to migration and changing wage structures. Our participants indicated that authority figures remained important, but this authority was negotiated. The notion of 'key decision makers' was used to identify an authority figure within the family who would act as a spokesperson. Whilst this role was depicted as a departure from traditional family models, a number of traditions remained. The maintenance of family harmony continued to be seen to be important and 'key decision makers' upheld this. That is, families would present a united front, making the type of open discussion favoured by therapeutic FIP models difficult.

There are a number of points that are relevant to clinical work. Mental health services within China are resource poor and most community staff have limited training. Consequently there is a need to consider whether staff believe FIP models can be effective. Our findings suggest that managers with limited mental health training would be willing to consider developing FIP as a community intervention, although they had some concerns about the utility of the model. From their perspective, FIP could only be delivered if families could be encouraged to engage over a period of time. Families in Guangzhou were seen as quick to withdraw from mental health services which can be understood as a response to group stigma and to scepticism in psychiatry. Managers believed that families would be most willing to accept FIP where an individual had an early diagnosis of psychosis. Whilst this view is in line with common practice internationally, it is unclear whether these families would engage for a longer period or whether the pressures of group stigma would be any less. The findings do seem to point to a need to incentivise FIP for families through linking it to concerns such as financial problem-solving. Results also suggest that whilst authority figures within families may be negotiated, traditional notions of family harmony remain, which may make therapeutic models of FIP challenging. Drawing on western models of FIP, which promote the expertise of the person with psychosis, is likely to be at odds with cultural practices affording the most influence to decision makers. This indicates that preference should be given to models focussing on interaction between carers in the first instance with service users being introduced to sessions at a later date. A model in which the person with psychosis is supported to avoid stress through continuing to recover within the family home is likely to fit better than a model promoting independence (as would be the case in western models of FIP) in a culture that values interdependence and parental authority. Whether families can be supported to move from seeing the person with a mental health problem as incapable or handicapped is not known. Future work will therefore need to focus on 
how the evidence base for FIP may be adapted so that it is culturally valid through engagement with keystakeholders such as people with mental health problems, carers and professionals.

A number of limitations of the study should be noted. This focus group was conducted as a pilot study and so the scale of the project was small. Consequently the emerging themes have not been tested against those of other mental health managers, although our sample did draw on the views of the majority of managers of IMHCs in Guangzhou. The city in which the research was based has a mental health service that is relatively developed. However, levels of service vary amongst provinces with provision in rural areas remaining particularly sparse (Wong et al, 2014). It is therefore possible that managers in different regions may reach other conclusions. Whilst managers gave accounts of how families might engage with FIP, the accounts of people with mental health problems and their carers may be different from these which may be a fruitful area for further qualitative work.

\section{CONCLUSION}

Policy makers in China aim to extend community based services which engage with families. FIP can offer an effective way to provide community support in a culture which has always placed an emphasis on the centrality of family. However, for the model to be effective attention needs to be paid to the way in which families with mental health problems may experience stigma. Means also need to be developed to maximise engagement within early sessions. To do this FIP sessions may need to focus around family concerns such as financial pressures. Further work may need to be carried out separately with carers and the person with psychosis in the first instance. China continues to go through rapid cultural change so attention needs to be given to the evolving nature of family dynamics within regions of China. 


\section{REFERENCES}

Braun, V. \& Clarke, V. (2006) Using Thematic Analysis in Psychology.Qualitative Research in Psychology, 3, 77-101.

Brown, G. W., Carstairs, G. M., \& Topping, G. (1958) Post-hospital adjustment of chronic mental patients.The Lancet, 272, 685-689.

Bucci, S., Berry, K., Barrowclough, C., \& Haddock, G. (2016) Family interventions in psychosis: A review of the evidence and barriers to implementation. Australian Psychologist, 51, 62-68.

Chakrabarti, S. (2011) Family interventions in schizophrenia: Issues of relevance for Asian countries. World $J$ Psychiatry, 1, 4-7.

Chao, R. K., \& Sue, S. (1996) Chinese parental influence and their children’s school success: A paradox in the literature on parenting styles. In S. Lau (Eds.), Growing up the Chinese way: Chinese child and adolescent development, (pp. 93-120). Hong Kong: The Chinese University Press.

Chien, W. T., Chan, S., Morrissey, J., \& Thompson, D. (2005) Effectiveness of a mutual support group for families of patients with schizophrenia. Journal of advanced nursing, 51, 595-608.

Falloon, I., Boyd, J. and McGill, C. (1984)Family Care for Schizophrenia. New York: Guildford Press.

Glynn, S. M., Cohen, A. N., Dixon, L. B., \& Niv, N. (2006) The potential impact of the recovery movement on family interventions for schizophrenia: opportunities and obstacles. Schizophrenia Bulletin, 32, 451-463.

Goffman, E. (1990) Stigma: Notes on the Management of a Spoiled Identity. London: Penguin.

Lam, P. C., Ng, P., Pan, J., \& Young, D. K. (2015) Ways of coping of Chinese caregivers for family members with schizophrenia in two metropolitan cities: Guangzhou and Hong Kong, China. International Journal of Social Psychiatry, 61, 591-599.

Li, X., Zhang, W., Lin, Y., Zhang, X., Qu, Z., Wang, X., Zhang, Y., Xu, H., Zhao, S., Li, Y. and Tian, D. (2014)Pathways to psychiatric care of patients from rural regions: A general-hospital-based study. International Journal of Social Psychiatry, 60, 280-289.

Li, Y., \& Chui, E. W. (2016) Community care for adults with severe mental disorders in rural China: A case study. International Journal of Social Welfare, 25, 273-282.

Livingston, J. D., \& Boyd, J. E. (2010)Correlates and consequences of internalized stigma for people living with mental illness: A systematic review and meta-analysis. Social science \& medicine, 71, 2150-2161.

McFarlane W.R. (2016) Family interventions for schizophrenia and the psychoses: A review. Family Process, 44, 460-482. 
Montgomery, W., Liu, L., Stensland, M. D., Xue, H. B., Treuer, T., \& Ascher-Svanum, H. (2013) The personal, societal, and economic burden of schizophrenia in the People's Republic of China: implications for antipsychotic therapy. ClinicoEconomics and Outcomes Research, 5, 407-418.

National Health and Family Planning Commission of China (2015)The National Mental Health Work Plan 20152020. Retrieved from http://www.gov.cn/zhengce/content/2015-06/18/content_9860.htm

Pearson, V., Paul, C., \& Lam, W. (2002) On their own caregivers in Guangzhou, China. In H. Lefley \& Johnson, D. (Eds.), Family interventions in mental illness: International perspectives, (pp. 171-184). Westport: Greenwood Publishing Group.

Pharoah, F., Mari, J., Rathbone, J., \& Wong, W. (2010) Family intervention for schizophrenia. Cochrane Database of Systematic Reviews, 12.

Phillips, M. R., Pearson, V., Li, F., Xu, M., \& Yang, L. (2002) Stigma and expressed emotion: a study of people with schizophrenia and their family members in China. The British Journal of Psychiatry, 181, 488-493.

Phillips, M. R., Li, Y., Stroup, T. S., \& Xin, L. (2000) Causes of schizophrenia reported by patients’ family members in China. The British Journal of Psychiatry, 177, 20-25.

Phillips, M. R. (1993) Strategies used by Chinese families coping with schizophrenia, In, D. Davis \& S. Harrell (Eds.), Chinese families in the post-Mao era, (pp. 171-184). Berkeley: University of California Press.

Pitschel-Walz, G., Leucht, S., Bäuml, J., Kissling, W., \& Engel, R. R. (2001) The effect of family interventions on relapse and rehospitalization in schizophrenia: a meta-analysis. Schizophrenia Bulletin, 27, 73-92.

Ramsay, G. (2010) Mainland Chinese family caregiver narratives in mental illness: Disruption and continuity. Asian Studies Review, 34, 83-103.

Ryan, K. E., Gandha, T., Culbertson, M. J., \& Carlson, C. (2014) Focus Group Evidence Implications for Design and Analysis.American Journal of Evaluation, 35, 328-345.

Sin, J., Gillard, S., Spain, S., Cornelius, V., Chen, T., \& Henderson, C. (2017) Effectiveness of psychoeducational interventions for family carers of people with psychosis: A systematic review and meta-analysis. Clinical Psychology Review, 56, 13-24.

Singh, S. P., Harley, K., \&Suhail, K. (2013) Cultural specificity of emotional over-involvement: a systematic review. Schizophrenia bulletin, 39, 449-463.

Smith, G., Gregory, K. and Higgs, A. (2007) An Integrated Approach to Family Work for Psychosis. A Manual for Family Workers. London: Jessica Kingsley Publishers. 
Wong, D. F. K., Zhuang, X. Y., Pan, J. Y., \& He, X. S. (2014) A critical review of mental health and mental health-related policies in China: More actions required. International Journal of Social Welfare, 23, 195204.

Yang, L. H., \& Kleinman, A. (2008) 'Face’ and the embodiment of stigma in China: The cases of schizophrenia and AIDS. Social science \& medicine, 67, 398-408.

Zubin, J. and Spring, B. (1977) Vulnerability: a new view of Schizophrenia. Journal of Abnormal Psychology, 86, 103-126. 